\title{
Metabarcoding analysis of the bacterial and fungal communities during the maturation of Preparation 500 , used in biodynamic agriculture, suggests a rational link between horn and manure.
}

\author{
Marina Zanardo ${ }^{1}$, Matteo Giannattasio ${ }^{2}$, Gaurav Sablok ${ }^{3}$, Massimo Pindo ${ }^{3}$, Nicola La Porta ${ }^{3,4}$, \\ Michele Lorenzetti ${ }^{5}$, Carlo Noro ${ }^{5}$, Piergiorgio Stevanato ${ }^{1}$, Giuseppe Concheri ${ }^{1}$ and Andrea \\ Squartini ${ }^{* *}$ \\ ${ }^{1}$ Department of Agronomy, Animals, Natural Resources and Environment - DAFNAE. University of Padua, \\ Viale dell'Università 16, 35020 Legnaro (Padova), Italy; marinazanardo1@gmail.com (M.Z.); \\ stevanato@unipd.it (P.S.); giuseppe.concheri@unipd.it (G.C.); squart@unipd.it (A.S.) \\ ${ }^{2}$ Fundación Emys, ctra. Santa Coloma 12, 17421 Riudarenes, Gerona (Spain); m.giannattasio@libero.it \\ ${ }^{3}$ Research and Innovation Centre, Fondazione Edmund Mach (FEM), Via Mach 1, 38010 S. Michele all'Adige, \\ Trento, Italy; sablokg@gmail.com (G.S.); massimo.pindo@fmach.it (M.P.); nicola.laporta@fmach.it (N.L.P.) \\ ${ }^{4}$ MOUNTFOR Project Centre, European Forest Institute, Via E. Mach 1, 38010 San Michele all'Adige, Trento, \\ Italy; nicola.laporta@fmach.it (N.L.P.) \\ ${ }^{5}$ Associazione Culturale Professione Biodinamica, Via Valle Fredda 31, 00030, Labico (RM), Italy; \\ miklorenzetti@libero.it (M.L.); carlo.noro@alice.it (C.N.).
}

*Correspondence: squart@unipd.it; Tel. ++39 0498272923

\begin{abstract}
Horn manure (Preparation 500) is a product used in the practice of biodynamic agriculture. It is obtained by an underground fermentation of cow faecal material incubated in cow horns for several months. The product is used as spray treatment meant to increase soil fertility. In the present report we analyzed the successional changes in bacterial and fungal communities throughout the process of horn manure maturation by high throughput sequencing of ribosomal 16S (bacterial) and ITS (fungal) gene markers. Marked shifts in the microbial community were seen involving a general decrease from a Firmicutes-dominated material to a product transiently enriched in Proteobacteria and later in Actinobacteria, mostly within the Nocardioidaceae family. In the fungal community evolution, the most abundant taxon in the starting faecal material resulted a member of the Onygenales order, known to specifically degrade keratin. Its abundance in the intestine is explained by the fact that keratin, which is also the structural component of hairs and horns, is found in all epithelial layers, including gut mucosae. This occurrence suggests a link of enzymatic/catabolic nature between manure and horn.
\end{abstract}

Keywords: Preparation 500; horn manure; biodynamic agriculture; keratin; Onygenales

\section{Introduction}


Organic farming includes several schools of thought, some of which follow protocols which are often based on popular traditions or doctrines that are drawn from empirical knowledge. The concepts on which these practices are based tend to be regarded skeptically as they apparently lack a clear scientific basis. Moreover, some aspects of the prescribed protocols appear overtly outlandish. One of these concerns the burial for 5-6 months of fallen cow horns after filling them with cowpats or finely ground quartz crystals to obtain the two field spray products named preparation 500 (cow horn manure) and preparation 501 (cow horn silica), which are used in biodynamic agriculture (BD), a practice developed in the 1920s by Rudolf Steiner [1]. Upon a dilution in water, these two preparations are subsequently used in the belief that they foster soil fertility and plant growth, respectively [2,3]. Although their real effectiveness and mode of action are being still debated, these preparations are featured in the list of materials and techniques officially allowed in organic farming by EC Regulations 834/2007 and 848/2018. Biodynamic farmers report as common practice the fact that, as regards horn manure, the horns can be used for 4-5 seasons and then discarded as their shell becomes progressively thinner (C. Noro, Personal Communication).

Different reports $[4,5]$ have claimed a positive effect of $\mathrm{BD}$ as concerns soil quality parameters including organic matter accumulation (carbon stocking), soil microbial biomass and its diversity. Those studies were based on a long-term field experiment comparing BD with standard organic farming and conventional management. The fact that the difference between biodynamic agriculture and plain organic farming rests essentially in the use of the BD preparations, stimulated research to verify whether and to which extent would such products really work, and to understand their possible principles of functioning. Literature reports have claimed that field treatments with the two preparations enable higher lentil yield and higher nitrogen content in wheat and soil, as well as higher nitrogen and microbially-available soil carbon [6,7]. In other studies instead the BD management was not reported to enhance soil fertility; a comparison of 10 conventional farms with 10 that had practiced biodynamics for 16 years reported that soil Olsen-extractable P concentrations were up to three-fold higher under conventional management and that there were few differences in soil biological properties, with earthworms weight significantly higher in the conventional farms, and no difference in soil organic carbon, nor microbial biomass [8]. In addition, it was observed that cow milk production was up to $36 \%$ higher in conventional farms and that parasitic infections had the same incidence under both conditions [9]. In terms of diversity and composition of the soil microbial communities, comparing in this case biodynamic vs. organic farming, no significant differences were detectable [10]. The same authors, focusing on the harvested products, reported that BD lettuce showed lower yields than conventional one but higher polyphenol content [10]. A different report analyzing polyphenol and biogenic amines content in grape berries from conventional, organic, and biodynamic vineyards could not find significant differences related to any of these managements [11]. Moreover, attempts to unravel the underlying mechanism of action of these preparations have often been inconclusive or contradictory; for reviews see: $[3,12]$. An explanation might be that, at present, it is still not possible to standardize these products because their chemical composition and their exact biological activity (if any) are still poorly known. Consequently, the quality of BD preparations, coming from different farms and years, could vary from one study to another and the results obtained could be not validly comparable.

In a prior study [13] we have investigated the composition of the horn manure preparation from a chemical standpoint using solid-state nuclear magnetic resonance spectroscopy and off-line TMAH-thermochemolysis-GC-MS. The molecular inventory showed that, while the hydrophobicity was similar to that of a plant-derived compost, the lignin components of the horn manure were more abundant and diverse. The presence of stabilized lignin derivatives, unlike the case of aboveground composting, was compatible with the slow, oxygen-poor underground fermentation process. NMR peaks were detected corresponding to several soluble and easily degradable compounds, including short chain fatty acids of microbial origin, free aminoacids, both of which qualify as potential microbial growth stimulators [13]. In addition, phenolics with antioxidant and growth regulating properties were detected. In our subsequent report [14] we analyzed several batches of horn manure 
from different farms focusing on biological properties as the quantitative assessment of the plateculturable microbial population, enzymatic activities and other phenotypes in the plant-microbe signalling repertory. The material was shown to possess high levels of $\beta$-glucosidase, alkaline phosphatase, chitinase, and esterase activities. The preparation had no quorum sensing-detectable signal, no rhizobial nod gene inducing properties but displayed a strong auxin-like effect on plants.

These properties indicate the product as endowed with a certain bioactive potential as soil biostimulant in fertility and nutrient cycling contexts. However, in our work we have not examined under any experimental framework what could be the actual outcome of its application in the field.

In the same paper we also dealt with an aspect that constitutes an element of criticism towards biodynamics practices. As the dosage involves a dilution of $200 \mathrm{~g}$ of hornmanure in 70 liters of water to be sprayed over a hectare surface, the practice had been judged as pertaining more to the domain of homeopathy than to that of agriculture. To verify whether the argument were oversimplistic, we calculated the actual molar dilution of the potentially active compounds by taking into account the total water-filled pore space of the hectare at plant roots depth, and showed that the final concentrations would range between $10^{-9} \mathrm{M}$ and $10^{-6} \mathrm{M}$. Many compounds are known to be active at far lower molarity; e.g. rhizobial chito-lipo oligosaccharides, triggering legume nodulation, start their activity at concentrations as low as 0.1 nanomolar $\left(10^{-10} \mathrm{M}\right)$ [15]. Furthermore, many hormones in biology act even at femtomolar concentrations $\left(10^{-15} \mathrm{M}\right)$. Therefore, if any active molecules were present in preparation 500, their delivery to the field appears compatible with a final concentration within the range known to have effect for other biostimulants.

In the present manuscript we focused on the preparation 500 following the total microbial populations by a DNA-based culture-independent molecular analysis of the bacterial and fungal components from the initial faecal material throughout its maturation to horn manure. The sampling schedule involved also two intermediate time points at which we pre-maturely dug out horns from the ground to have details on the ongoing community dynamics at different stages.

For what concerns the biodynamic doctrine in broad sense, we also feel necessary to state a relevant disclaimer; our studies are focused on single aspects of defined processes that are amenable to analytic investigation, leaving out (and not being prejudiced by) a number of eccentric aspects that accompany the biodynamic tradition, as those invoking cosmic forces or anything non-measurable by current methodologies.

Likewise, all conclusions we provide are to be seen as relative to the single piece of context that we analyze, sticking to hard facts and not projecting beyond their evidence. Therefore, a description of the microbial communities within the preparation 500 is not to be mistaken as supporting the efficiency or the advantages of biodynamic agriculture, which would have to be tested by the appropriate rigorous trials.

\section{Materials and Methods}

\subsection{Horn manure preparation and sampling schedule}

The production of horn manure was carried out at "Società Agricola Biodinamica" (Labico, Rome), which is a long since established biodynamic farm from which also some of the materials used in earlier surveys had been obtained [13,14]. The forage by which the manure-producing cows are fed is also grown in the same farm and cyclically delivered to its soils.

Analyses at each time point were done on three full biological replicates. The initial faecal material prior to introduction into horns constituted the time zero samples; followed by two intermediate sampling times at which we extracted three horns, at day 40 and three at day 85, while the last sampling occurred at day 150, corresponding to the final maturation of the analyzed product.

Additionally, the mature sample (150 days) was split in two parts. The inner core material at the center of the horn, and the outer layer close to the horn shell, which were analyzed separately. The sampling was 'destructive' i.e. for each subsequent sampling time, three different horns were 
excavated. Either the whole horn content, or the inner and outer portions (for the samples at 150 days) were homogenized prior to the subsampling of aliquots for DNA extraction. Samples were air-dried at room temperature in thin layers over pleated paper prior to proceed with DNA extraction. We also analysed the soil of the same farm, which represents the environment which yearly receives the horn manure applications. Soil was cored at the surface within the first $10 \mathrm{~cm}$ layers and air-dried as described above for the other samples. Three replicates were taken also for the soil samples.

\subsection{DNA Extraction, construction of the gene libraries, and pyrosequencing}

Genomic DNA was extracted from each sample using the QIAamp DNA Stool Kit (Qiagen, Milano, Italy) following the manufacturers' instructions, and its quality assessed by gel electrophoresis and the NanoDrop spectrophotometer (Thermo Fisher, Waltham, MA) and stored at $-20{ }^{\circ} \mathrm{C}$ until pyrosequencing.

For the bacterial communities, we investigated the 16S rRNA gene using the primer set specific for the V1-V3 hypervariable regions (27-Forward: 5'-AGAGTTTGATCMTGGCTCAG-3' [16] and 533Reverse: 5'-TTACCGCGGCTGCTGGCAC-3' [17].

For the fungal communities, the ITS rDNA gene was analysed using the primer set specific for the ITS1 hypervariable regions (ITS1-F primer: 5'-CTTGGTCATTTAGAGGAAGTAA-3' and the ITS2 primer 5'-GCTGCGTTCTTCATCGATGC-3' [18]. The forward primer contained the universal Roche/454 "Lib-L" primer A, the key sequence (TCAG) and the barcode (Multiple IDentifier) sequence (specific for any sample). The reverse primer contained the universal Roche/454 "Lib-L" primer B and the key sequence.

The PCR products, obtained using the FastStart High Fidelity PCR system (Roche Life Science, Milano, Italy), were analyzed by gel electrophoresis and cleaned using the AMPure XP beads kit (Beckman Coulter, Brea, CA, USA) following the manufacturer's instructions. Subsequently amplicons were quantified via quantitative PCR using the Library quantification kit Roche 454 titanium (KAPA Biosystems, Boston, Ma) and pooled in an equimolar way in a final amplicon library. The high throughput sequencing was carried out on the GS FLX+ system using the XL+ chemistry following the manufacturer's recommendations.

\subsection{Bioinformatics}

454 bacterial and fungal reads were cleaned for the primer and the MID (multiplex identifier) and reads shorter than $100 \mathrm{bp}$ and with quality score below 15 were discarded. Reads were denoised to correct for the sequencing errors using Amplicon Noise [19] and subsequently checked for chimeras. After chimeras removal reads were analyzed for the identification of the appropriate regions. Both chimera cleaning and identification of the bacterial and the fungal OTUs were done using QIIME [20] version 2.2019.7. The filtering steps were followed by subsampling to 4217 randomly selected $16 \mathrm{~S}$ bacterial sequences and 6364 ITS fungal sequences per sample to correct for differences in sequencing depth. For the fungal sequencing, classification of the OTUs was done using UNITE [21] and INDSC (https://www.ncbi.nlm.nih.gov/genbank/collab/) fungal specific databases. For the bacterial sequencing, OTUs were defined using the SILVA (Release 132) and RDP (release 11) databases. Identified OTUs were classified according to the phylum, class, order, family and genus. Species richness and alpha - and beta- rarefaction analysis were done using the QIIME pipeline [20]. Raw data were deposited in the EMBL-EBI public database (https://www.ebi.ac.uk/) where they are accessible under code PRJEB25151. 


\subsection{Statistical analyses}

The statistical significance of differences among samples was explored first by running Principal Coordinate Analyses (PCOA), to which the PERMDISP2 analysis of multivariate homogeneity of group dispersions (variances) with an ANOVA test was subsequently applied. The PEERMDISP2 tool is implemented in $\mathrm{R}$ using the betadisper function (package vegan, version 2.4-2, License: GPL-2). For the above analyses the data used were the total sum of squares (TSS) with square root transformation of the OTU table abundances matrix. All these analyses were carried out by the Calypso online utility (http://cgenome.net/calypso/). The PAST Software (https://folk.uio.no/ohammer/past/) was used to calculate species diversity/evenness indexes, Whittaker beta diversity distances, and cluster analysis ordination trees, using the plain OTU table matrix.

\section{Results}

\subsection{Overall microbial community partitioning}

The run statistics reported a mean value of $77.5 \%$ of reads passing the quality filter, as regards the $16 \mathrm{~S}$ bacterial amplicons; the sequencing yielded a consequent throughput of 197463 reads, averaging 10970.17 reads per sample with a standard deviation of 3414.5 .

For the fungal ITS primers the run statistics reported $78.2 \%$ of reads passing the quality filter. The sequencing yielded a consequent throughput of 214232 reads, averaging 11901.7 reads per sample with a standard deviation of 2715.5. All samples met the expected standards of saturation upon inspection of the rarefaction curves for both sets of primers.

In terms of community richness, a minimum of 44 and a maximum of 99 genera of fungi per sample were observed depending on the maturation stage while the corresponding values for bacteria ranged from 60 to 172. Data dynamics and their variability for the two groups of microorganisms are shown in Fig. 1. Numerical details are displayed in Table S1 (Supplementary material). The values of the soil of the same farm from which the manure is produced and on which the matured preparation 500 is applied are also shown, which spanned from 96 to 108 genera for fungi and from 193 to 202 for bacteria. 


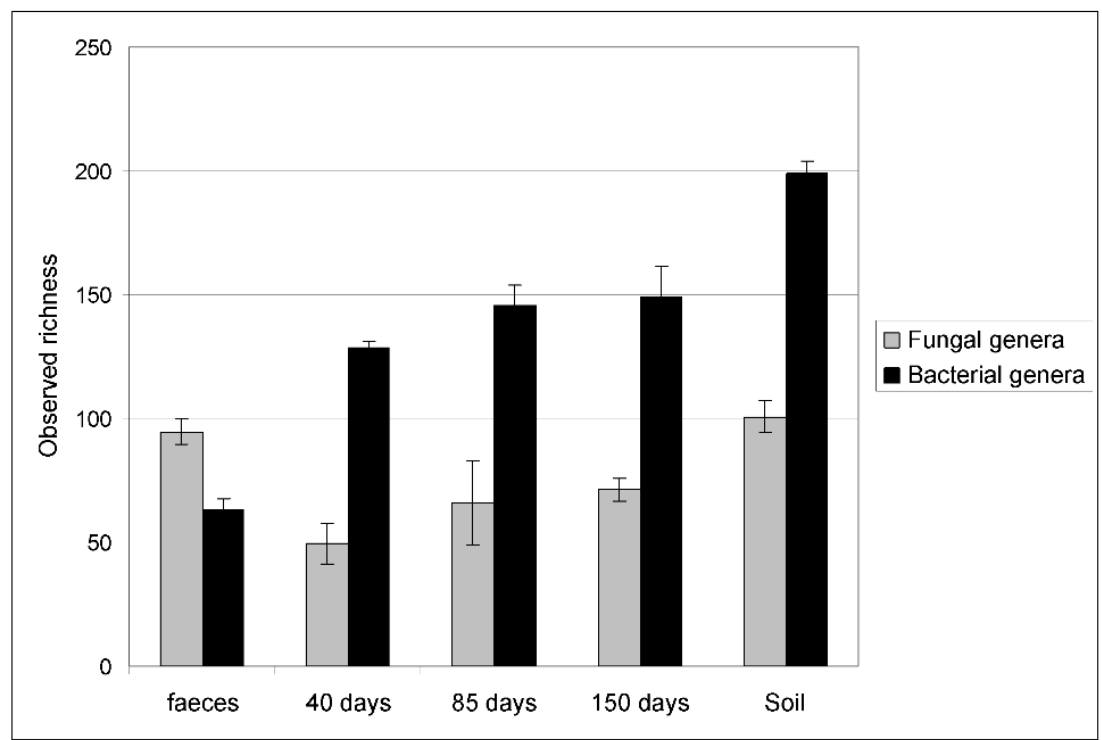

Fig. 1. Dynamics of the bacterial and fungal richness within the buried horns throughout the maturation process. The mean and standard deviation of the three replicates are shown. For the samples at 150 days the mean includes values of the inner and outer layer.

Statistically significant differences across communities observed at the different sampling times were examined by PERMDISP2. The procedure, which is an R-implemented algorithm, tests if global community composition is significantly different between groups and visualizes the distances of each sample to the group centroid in PCoA providing a p-value for the significance of the grouping. Data showed, for both $16 \mathrm{~S}$ and ITS data, an overall significant difference which was more pronounced for the bacteria ( $p$ Value $\left.=2.05 \mathrm{e}^{-05}\right)$ then fungi $(\mathrm{pValue}=0.00343)$ and a very clear separation of the different time points in the principal coordinate plots. Results are shown in Figs. 2, and 3 for bacteria and fungi, respectively. The consistency of data within replicates and the respective differences between each sampling are further appreciable by observing the beta diversity pairwise comparisons for the Whittaker distances of all communities for bacteria and for fungi, which are shown in supplementary figures S1 and S2 respectively. For the bacteria it can be seen that the most dissimilar communities are those of the manure from those of the soil and that a gradient of decreasing similarity characterizes the maturation stages as can be seen by a vertical inspection of the first three columns. For fungi instead, the situation shows less unidirectional trends, a slight similarity between the manure and the final stage after a drift in the intermediate stages. The 40-day fungal community resulted the least similar to the soil one. The relative resemblance of the communities and the variability among replicates can be independently seen in the two Neighbor Joining dendrograms obtained from the Bray Curtis dissimilarity data, shown in supplementary Figs. S3 and S4 for the bacterial and fungal data respectively. In these it can be appreciated that all triplets of replicates form coherent clusters with shorter distances among themselves than from the different treatments. The ecological indexes for richness and evenness of all communities are shown in Supplementary Table S2. These show that bacterial diversity and evenness peak at 85 days to slowly decrease later. Fungi, besides their constantly lower values, show instead a drop at 40 days and a subsequent steady rise along the material maturation. 

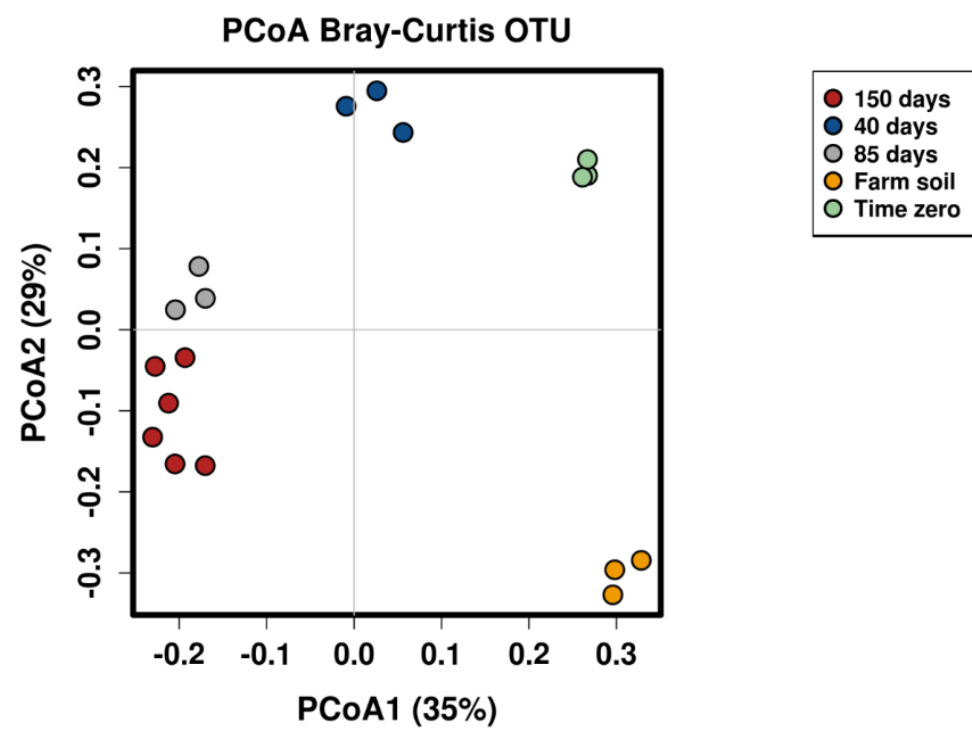

Fig. 2. Principal Coordinates Analysis based on the Bray Curtis dissimilarity of the bacterial (16S) data. Samples at 150 days include both inner and outer core cases. Upon running a PERMDISP2 analysis of multivariate homogeneity of group dispersions (variances), the ANOVA test based on the Principal Coordinates analysis scored a significant $\mathrm{P}$ value equal to $2.05^{\mathrm{e}-05}$

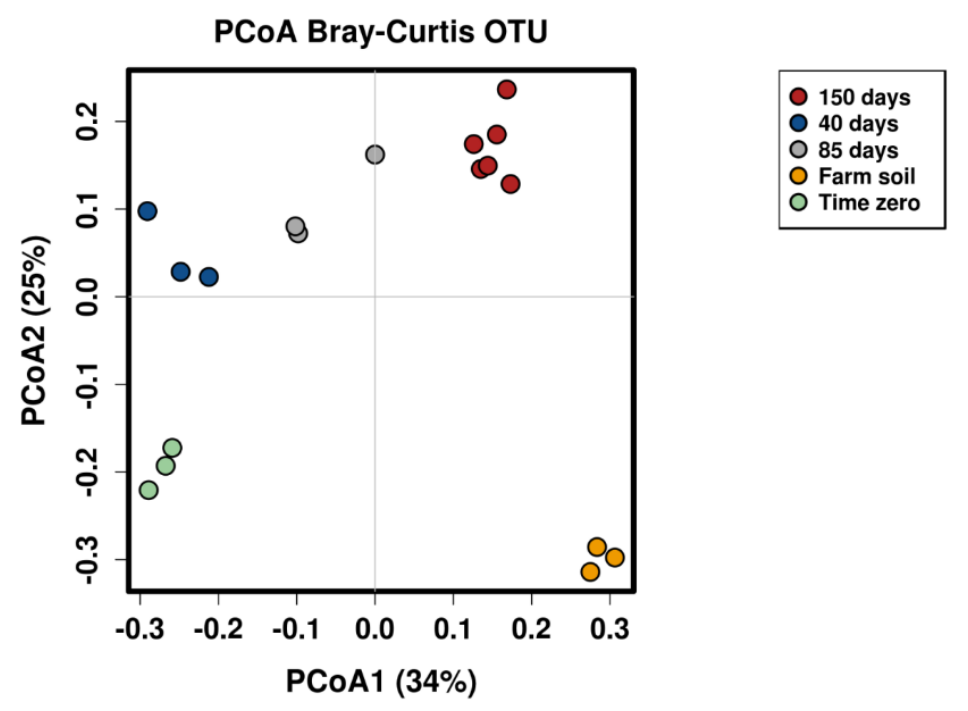

Fig. 3. Principal Coordinates Analysis based on the Bray Curtis dissimilarity of the fungal (ITS) data. Samples at 150 days include both inner and outer core cases. A PERMDISP2 analysis of multivariate homogeneity of group dispersions (variances) with an ANOVA test, based on the Principal Coordinates analysis scored a significant $P$ value of 0.00343

\subsection{Bacterial taxonomy}

As regards the qualitative shifts of the different taxonomical groups, Fig. 4 shows the substitutional dynamics of the bacterial component at phylum level. Substantial community substitutions occur that involve the relative depletion of Firmicutes on one side, paralleled by the progressive increases of Proteobacteria, and mostly of Actinobacteria, that dominate the mature stages, along with instances of Cyanobacteria, Lentispherae and Acidobacteria. 


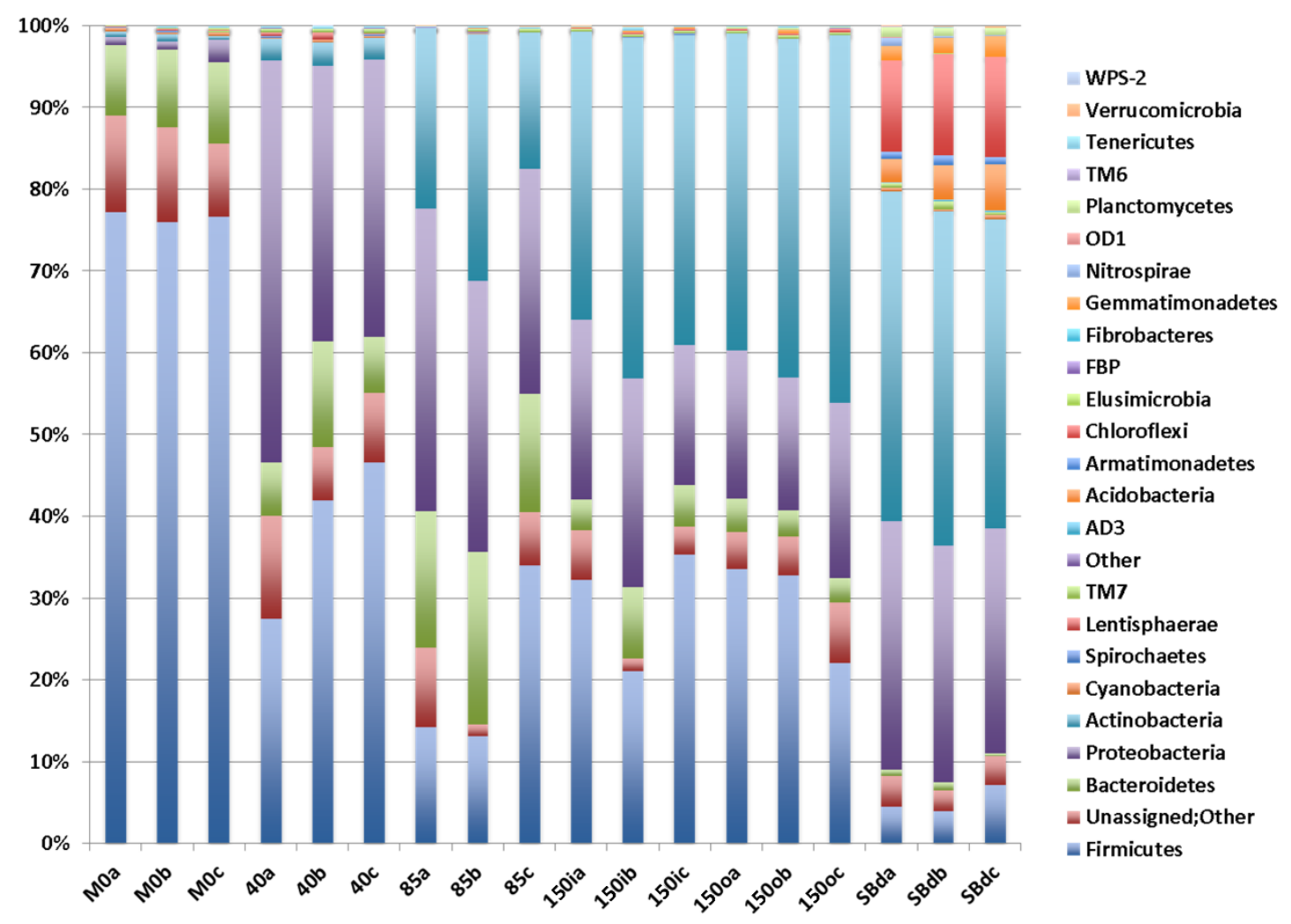

Fig. 4. Bacterial phyla observed and their relative percent contribution to each timepoint community and to the soil of the project's farm (SBd). The terms, assigned by the annotation pipeline, correspond to the officially referenced taxa whose details can be browsed in the NCBI database at the address: https://www.ncbi.nlm.nih.gov/Taxonomy/Browser/wwwtax.cgi

The same communities analysed at the deepest systematic level achievable through comparison to the database annotations (genus or family or order depending on the presence of existing subject records), provide further systematics details. The data concerning the 20 most abundant taxa (ranking the list by their mean abundance across all samples) are reported in Supplementary Information Fig. S5, in which the identities of the bacteria whose phyla were presented in Fig. 4 are detailed. It can be seen that the dominance of manure by Firmicutes is represented mostly by the Clostridiales and to minor extent by Bacillales orders. Meanwhile, in the mature product, the Actinomycetescharacterized community is defined in large part by the order Actinomycetales and in particular by the family Nocardioidaceae. The intermediate stage (40 days) features a major transient dominance of the genus Acinetobacter, which almost completely fades out later.

\subsection{Fungal taxonomy}

Coming to the analysis of eukaryotic fungal biota, sequencing of the amplicons raised by the ITS primers allowed to determine the situations depicted in Fig.5 and Supplementary Information Fig. S6. The former reports the finer resolution evidencing the 20 most abundant OTUs set at genus level while the latter shows the picture from an high-rank taxonomy level (classes). The Eurotiomycetes taxon abundant in faeces is a member of the Onygenales, an order typically involved in keratin degradation, while the member of Leotiomycetes that leads the scores until 


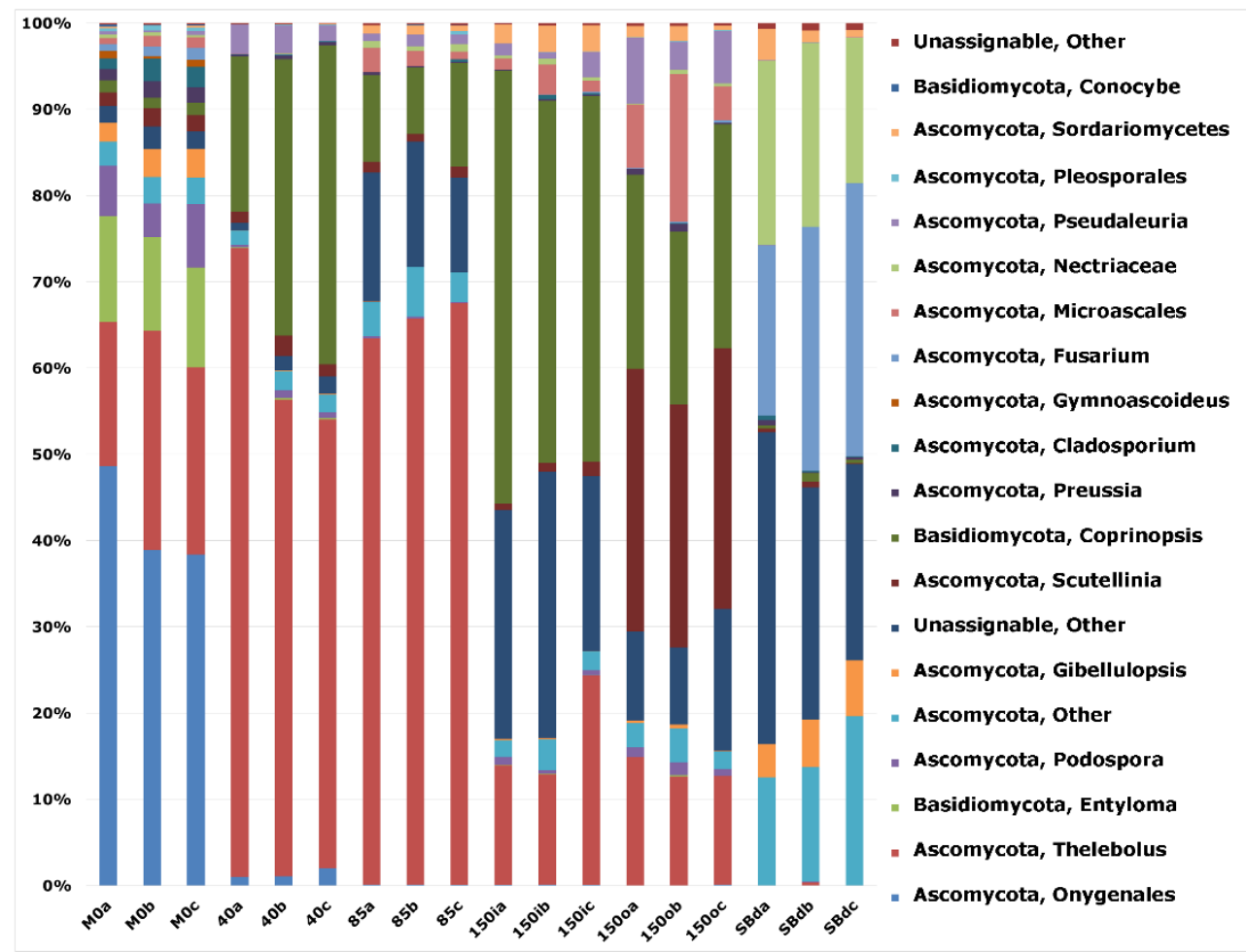

Fig. 5. Fungal genera, first 20 most abundant taxa. The nomenclature reported shows the Class followed by the deepest rank (L6) achievable by the annotation procedure. For details on the annotated names, the same web reference indicated in the legend of Fig. 4 applies.

day 85 is genus Thelebolus. The abundant Agarycomycota characterizing the mature preparation is ascribed to genus Coprinopsis.

The genus Scutellinia, according to metagenomics ITS sequencing resulted instead the most abundant genus in the outer core at final stages of maturation. As the fruiting bodies of this fungus are normally easily visible in nature, it was possible to seek their presence also by visual inspection on the surface of the horn manure after extraction from the horn shell. Its appearance is shown in Fig. 6, where the surface presents several whitish round-shaped efflorescences, most of which forming clusters. Upon scraping off material from the discs' upper face and observing it under light microcopy, the material revealed the abundant presence of ellipsoid bodies whose size and shape are compatible with those reported for the Scutellinia ascospores.

A further typical visual indicator of a soundly accomplished preparation, as reported by biodynamic practitioners, is a small $(2-6 \mathrm{~mm})$ fungus, round-bodied at that stage, with a black surface and a white flesh. Specimens of that were provided by the Labico biodynamic farm (Fig. 7) and pieces of mycelium were analyzed using the same ITS1 region. A single sequence with a $99 \%$ identity to Coprinopsis spp. was obtained.

As regards the overall experimental design, concerning the number of replicates used (three) and its appropriateness to correctly describe community composition, we have addressed the aspect assessing the extent of data dispersion around the mean by calculating the standard deviation of the three replicates; we then obtained the coefficient of variation (SD/mean) for each taxon found in all replicates in at least one sampling point; the $\mathrm{CV}$ values averaged 0.3 , which accounts for a satisfactory replication scheme. The adequacy of the set up was also confirmed by the contained variation within replicates shown by the PCoA (Figs. 2, 3) by the Whittaker beta-diversity distances (Figs. S1, S2) and by the coherency of clusters in NJ trees (Figs. S3, S4). 

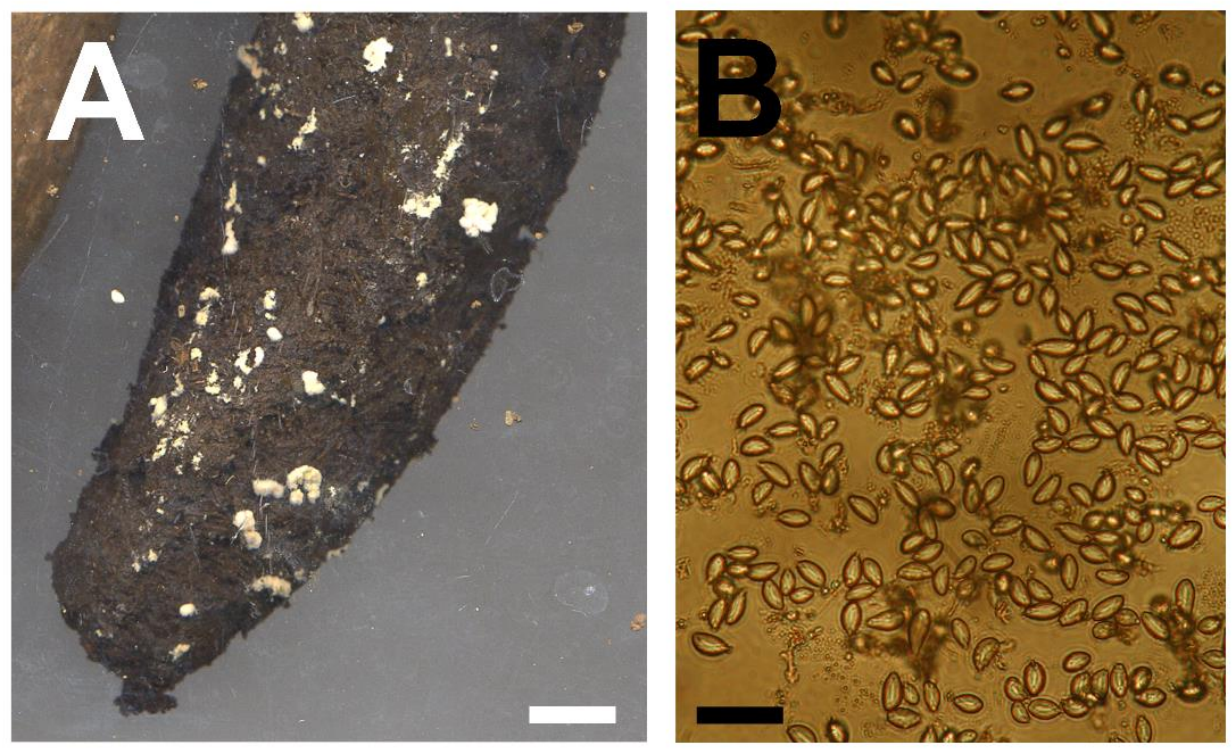

Fig. 6. Fungal efflorescences on mature material and their spores A: distal end of a horn manure cone casted out from the horn mould at day 150. B: brightlfield light microscopy image of material scraped off from the white discs visible in the left panel and resuspended in physiological solution. Scale bars: A: $1 \mathrm{~cm} ; \mathrm{B}: 50 \mu \mathrm{m}$.

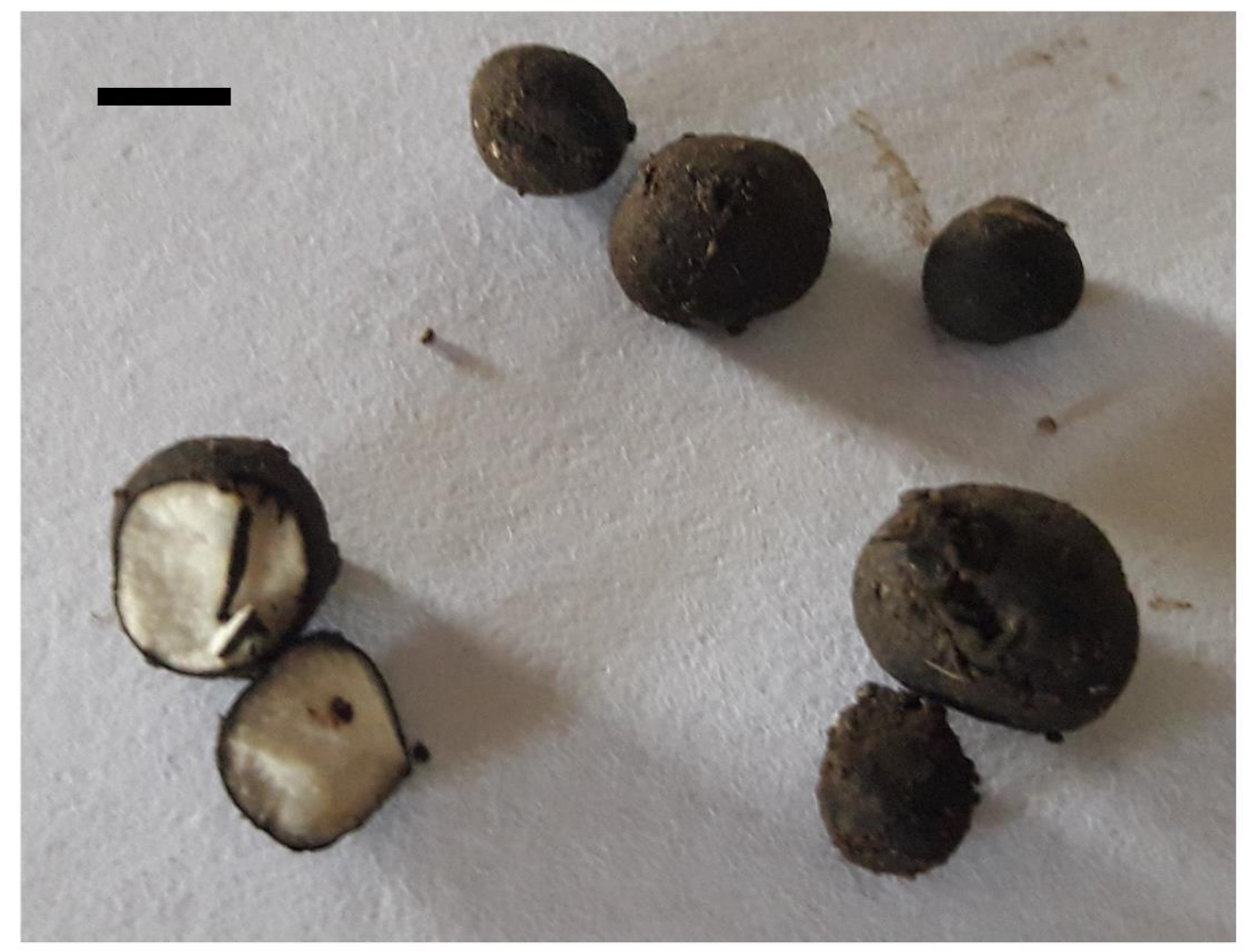

Fig 7. Fungal fruit bodies rescued from hornmanure prepared in year 2017 at the Labico biodynamic farm. The presence of these is reported as typical of a proper hornmanure preparation outcome. Resequencing of the ITS1 region from the mycelium of this material yielded a sequence with over $99 \%$ identity to Coprinopsis sp., in line with the metagenomics results where this genus was the dominant member of the inner core at day 150. 


\section{Discussion}

\subsection{General comparisons}

Following the dynamics of the number of genera through time (Tab. S1 (Supplementary material) and Fig. 1) it is possible to see that bacteria and fungi display an opposite behaviour. Fungal richness, was higher in the starting manure than at any of the subsequent stages and showed a minimum value at 45 days. On the contrary, bacterial diversity in quantitative terms showed a doubling of numbers when comparing the initial manure with the first sampling time, and a continuing increase in the later stages. The number of bacterial taxa was higher than that of fungal ones as regularly observed in general environmental studies, also in light of the different criteria adopted for species definition.

\subsection{Bacterial community dynamics}

While there are no prior literature reports on horn manure culture-independent metabarcoding, some indirect comparisons can be made. As concerns the starting cow manure, its bacterial community composition is in line with the profile known for bovine faecal material, featuring a majority of Firmicutes and a variable amount of Bacteroidetes as main taxa [22,23]. A major event observed in the early stage of maturation of manure in our study is the transient 'bloom' of the genus Acinetobacter (Proteobacteria) at 40 days (Supplementary Fig. S5). This member of the Gammaproteobacteria class had an average presence below $1 \%$ in the starting manure and reached a mean of $24 \%$ in the first five weeks of incubation where it dominated the community in all three replicates, to recede later to values below $0.1 \%$. Members of this genus are known as important soil inhabitants where they actively contribute to the mineralization of several forms of organic matter, including aromatic compounds [24]. The late and final stages are instead overwhelmingly characterized by two different Actinobacteria, both within the order Actinomycetales and the major of which represented by a taxon within the Nocardioidaceae family. These chemoorganotrophic organisms occur in various environments (soils, aquatic environments, plants) and some are active in the degradation of recalcitrant chemicals [25]. A further study that allows some comparisons with our analysis is a report on the changes that occur in open field in bovine defecated material [26]. In that case sampling took place for fresh cowpats for 57 days. In analogy with our case, authors also report the reduction of Firmicutes and an increase of Actinobacteria with Nocardioidaceae as prominent family, however they record also a major rise in Alphaproteobacteria (families Bradyrhyzobiaceae, Hyphomicrobiaceae, Rhizobiaceae, Phyllobacteriaceae) while in our case the only relevant Proteobacteria are from the Gamma- class (Acinetobacter and Pseudomonas) with a minimal presence of Alphaproteobacteria (Rhodobacteraceae) in the early stage. The differences between our results and those from the study of Wong and coworkers [26]. are explainable by the fact that cow pats in the field are exposed to air, sunlight, and direct deposition/inoculation of airborne and insect-borne immigrant microbiota, which could contribute to different successional dynamics. The taxa observed by those authors testify indeed a gradual shift from the anaerobic bovine gut to the aerobic pasture ground. Evaporating moisture from the cowpats and insect pitting, further foster the air-driven nature of those communities. In the buried cow horns instead, the environment is to be regarded as prevailingly microaerophilic and thus driving gradual maturation with a slow degradation of organic matter.

\subsection{Fungal community dynamics}

As regards the fungal component we have not found literature to compare these data as ours appears to be the first study dealing with metabarcoding of fungi in faecal material. Nevertheless the analyses at class and genus level, allow to infer some clear trends and a basic evidence. The fungus that mostly characterizes the time zero faecal material is a member of the Onygenales. Its striking overrepresentation in the time-zero manure in comparison to all other sampling points was statistically quantified to a $\mathrm{p}$ value significance of $7.8 \mathrm{e}^{-14}$ (Supplementary Fig. S7). This group is known to degrade keratin $[27,28]$, which is the key structural fibrous protein material making up hair, horns, claws, hooves and the outer layer of skin. The primary habitats of Onygenales (etymology is from ony $x=$ nail) are in fact animal skin and droppings. But it is worth remarking that keratin is also 
present in overall epithelia including the gut mucosae [29]. The nature of Onygenales as saprobionts on feathers, horns, hooves, or on soil and dung material has been reported [30].

The fact that this fungus results in all replicates the dominant member of the cow fecal material community (Fig. 5) is in our opinion a point worth of particular notice. The reason is not just the fact that its presence is consistent with stool being an epithelium-challenging material while keratin protects epithelia from injuries. Dislodged keratin-rich epithelial cells accompany the material and can sustain the growth of keratinolytic microbiota. The aspect that we find worth remarking is that in the horn-manure practice the scats are placed within the fallen horn. This operation joins a surface made of $100 \%$ keratin to a material which has been enriched with a specific keratin-degrading fungus throughout the whole intestinal transit.

The action of the keratin-degrader appears to unfold fast, as its relative frequency at 40 days is reduced by the increase of other fungi, among which one that was also present in faeces is Thelebolus, a genus known to include dung-inhabiting species whose evolution involved an alternate challenge: enduring the cold temperatures when growing on excreted faeces, but surviving the passage through warm-blooded animals gut [31]. This taxon is the second most abundant fungus in the faecal material, which becomes then the dominant in the horn manure stages at day 40 and day 85 sampling times.

It could be argued why the competence of the Onygenales fungus for keratin should not entail a massive increase of its share along the maturation. One answer is the fact that, while in the faecal material, mixed by peristaltic movements, its presence is expectedly homogeneous, its growth in the horn will be confined to the thin interface with the horn shell. As sampling was done by mixing homogeneously the material, a taxon growing on a specific surface would be diluted in the bulk material. Second, when a species performs a pioneering activity within a community (as scavenging amino acids from the horn surface and making those available also for the non-proficient members) it is often outcompeted in the subsequent stages by ordinary ecological successions. Third, when comparing communities at different time points with DNA-based methods it has always to be considered that the results are based on the calculation of the relative abundance of taxa (percent values). This implies that the growth of any, determines obviously a reduction of the relative abundance of others when those do not grow at equal or higher pace. This means that 'species A' could have been increasing, but its share in the sum could appear as if it had instead decreased if 'species B' has grown faster. This consideration, that applies inevitably for all metagenomics studies, should be kept in mind for all kinds of interpretations about increases and decreases which could be either real or apparent (when driven by a stronger change of a different group).

Other fungi present in the faeces but not conspicuously encountered in the subsequent stages of horn manure are Entyloma, a genus encompassing smuts [32], and Podospora, a filamentous, coprophilous herbivore [33].

At 150 days the inner and outer core of the horn manure had similar composition; however the inner portion showed nearly double values for the fungus Coprinopsis (Basydiomycota, formerly classified within the genus Coprinus) when compared with the outer layer, in which there was instead a trend of enrichment in genus Scutellinia (Ascomycota). The former, i.e. the dominant fungus in the inner horn manure core, is a mushroom of the ink-cap, shaggy mane group. These fungi are reported to fruit in fields where animal scats had been laid [34], hence the etymological root of the genus name (còros = dung). The genus Scutellinia, dominating the outer layer of the 150 day horn manure (Fig. 5) is a relative of the 'eyelash cup' type of fungi, a saprophytic group reported to occur on rotting organic matter and damp habitats, typically growing in small discoidal clusters reaching sizes from 1 to several millimeters. It is mostly distributed in the Northern hemisphere and 66 species are known [35]. This fungus morphology is consistent with the structures actually observed in the mature material (Fig. 6). The presence of these white spots on the surface of the horn manure when extracted from the horns is also reported by biodynamic farmers as a popular sign of proper maturation and expected satisfactory quality of the material's properties. We also inspected the black round macroscopic fungi that are characteristic of regular lots of the preparation (Fig. 7) and that gave a single sequence $99 \%$ identical to that of Coprinopsis sp. That occurrence gathered from the isolated tissue is in line with the above mentioned independent metagenomics results from the 
hornmanure total DNA, where Coprinopsis was in fact the fungal sequence resulting with the highest abundance in the inner core of the material at the final sampling date.

In addition to the visual clues, the other practical indicator of a complete process is the odor of the material, which should be that of a soil/compost-like substrate with no residual nuances of its faecal origins. In our time-lapse sampling we have had the confirmation of this as only the final 150 day samples had the neutral smell of the expected final material. Moreover, in the sampling at day 85 it was observed that the outer portion was already compliant to this property while the central core was not as dry and humus-like as the outer layer and still bore a stingy stool odor. This gives another information about the fact that the maturation process is centripetal. Therefore, the proteolytic action on the keratin horn shell exerted by fungi such as the cited Onygenales representative, appears to be a possibly important driver of the whole process. It can be commented that keratin is among the richest proteins in terms of sulphur content as its toughness is achieved by densely distributed disulphide bonds. The mineralization of $S$ and/or the availability of sulphurcontaining aminoacids and peptides could be therefore envisaged as important aspects of the process and possibly of the horn manure properties when sprayed in the field.

\subsection{Horn contribution to the process}

Regarding the importance of the horn itself in the manure transformation, we had previously tested (Giannattasio and Squartini, unpublished) whether maturation would be equally achieved in plastic cones instead of cow horns, or upon wrapping the stuffed cow horn in cellophane, either totally or leaving the base hole uncovered and vice versa (wrapping only the sides). Results showed that the only two combinations that allowed a full (odorless) maturation were either the regular cow horn or the version in which this was wrapped sideways with the circular hole open. Therefore, two aspects appear critical; a) the presence of the keratin horn that cannot be replaced by plastic, b) an inlet for air-moisture exchange. Although horns are placed in a $60-\mathrm{cm}$ deep pit covered by soil, the microaerophilic condition appears not substitutable by a tight anaerobic enclosure. The possible immigration of soil bacteria from the open end could also be a requirement in this respect.

As regards physical and chemical variables, notions on their variation during the process from fresh manure to preparation 500 are available in literature [36] and indicate a $\mathrm{pH}$ decrease from 7.8 to 5.6 , a moisture decrease from 84.3 to $72.9 \%$. Temperature fluctuations are considered to be minimal due to the underground burying condition.

The importance of the horn as the required shell for the process and the criticality of its conditions are also testified by Steiner's textbooks recommendations and by the observed practice to change and discard the horn after 4-5 uses. Consumption and thinning of the inner surface is regarded as the reason that prevents unlimited burial of the same piece. These aspects are central to the present work as they prove that the horn, which is for $100 \%$ made of keratin, is thence actually degraded during the process and that thus keratynolysis does occur. In this respect it can be hypothesized that the availability of a thick, sulphur-rich layer of keratin is a critical requirement for an efficient priming of the hornmanure maturation. The horns used in the present research were all within 1-3 times of usage as common practice of the hosting farm.

\subsection{Farm soil microbial composition}

Finally, a comparison can be made between the horn manure microbial composition and that of the soil of the same farm of production and cyclic redistribution (SBd samples). While a soil is a completely different environment and the horn manure is delivered annually at a very low ponderal rate (200 grams/hectar) some common taxa between the mature product and the soil are encountered. In particular these involve the bacteria of the Actinobacteria phylum, for which the main taxon in horn manure was the Nocardioidaceae family, which are involved, as most Actinobacteria, in organic matter processing and consequent nutrient cycling. These scored a mean around $18 \%$ in the horn manure and $1.85 \%$ in soil which is an unusually high value for this family in agricultural soils. However the hypothesis that such high numbers of Nocardioidaceae would be linked to the delivered hornmanure and to their capability of persisting/multiplying in the Labico farm remains to 
be demonstrated. Also for the other taxon within the Actynomycetales order ( $8.9 \%$ in horn manure) a strong presence in that soil (3.4\%) is observed.

Another evidence is that, within the Proteobacteria, the majority of sequences is represented by the family Rhodospirillaceae. Their group, also known as purple non-sulphur bacteria, can perform photosynthesis; they can photo-assimilate simple organic compounds under anaerobic conditions. They are also capable of chemotrophic growth under aerobic conditions; at oxygen concentrations between $0.5 \%$ and $5 \%$, their photosynthesis and oxidative metabolism may function simultaneously, and they are also known for their free-living nitrogen-fixing capability [37]. In general this composition is suggestive of a community with a high degree of autotrophy for carbon and nitrogen and able to convey a consistent flux of energy and matter through soil, which are indicative of a certain degree of habitat sustainability. Authors should discuss the results and how they can be interpreted in perspective of previous studies and of the working hypotheses. The findings and their implications should be discussed in the broadest context possible. Future research directions may also be highlighted.

\section{Conclusions}

In conclusion, the present study pointed out some possible catalytic connections between the main representative of the manure fungal community and the horn's composition. The dominance of a fungus with a tight ecological attitude, i.e. the capability of digesting keratin, suggests that horns, which are made of keratin, could be suitable substrates for a specific proteolytic decomposition process.

Reporting this evidence is nevertheless, not willing to confer any academic seal of endorsement or approval to the biodynamic practice, but merely to describe one of its preparations and comment on the data that arose upon analyzing its maturation. Any inference on the horn manure actual effects in crop productivity would require dedicated studies at field level to be carried out under the appropriate methodological framework.

Supplementary Materials: The following are available online at www.mdpi.com/xxx/s1, Figure S1: Whittaker beta diversity pairwise distance matrix of the bacterial community data., Figure S2: Whittaker beta diversity pairwise distance matrix of the fungal community data, Figure S3: Cluster analysis ordination of the bacterial data, Figure S4: Cluster analysis ordination of the fungal data, Table S1: Bacterial and fungal genera numbers recorded at different time points, Table S2: Ecological indexes of diversity and evenness, Figure S5: Bacterial first 20 most abundant taxa, Figure S6: Fungal classes observed, Figure S7: Onygenales fungi abundance comparison at different timepoints.

Author Contributions: Conceptualization, M.G., C.N., M.L. and A.S.; methodology C.N., M.L. M.G. and A.S.; investigation, M.Z., G.S. and M.P.; formal analysis, G.S., A.S. and M.P.; validation, A.S., N.L.P., G.C. and P.S.; writing - original draft preparation, A.S.; writing - review and editing, M.G., N.L.P. and A.S.

Funding: This research received no external funding.

Acknowledgments: Carlo Noro and Michele Lorenzetti, whose activity is on the side of horn manure making and which hosted the research at farm level, are acknowledged for having accepted the invitation to become also co-authors of the present manuscript, and, in doing so, upon accepting the responsibility of underwriting it, to share the consequent neutral and objective position in the analysis and interpretation of the data.

Conflicts of Interest: The authors declare no conflict of interest. 


\section{References}

1. Koepf, H.; Pettersson, B.; Schaumann, W. Biodynamic Agriculture; The Anthroposophic Press: Spring Valley, New York, 1976;

2. Carpenter-Boggs, L.; Kennedy, A.C.; Reganold, J.P. Organic and Biodynamic Management Effects on Soil Biology. Soil Sci. Soc. Am. J. 2000, 64, doi:10.2136/sssaj2000.6451651x.

3. Turinek, M.; Grobelnik-Mlakar, S.; Bavec, M.; Bavec, F. Biodynamic agriculture research progress and priorities. Renew. Agric. Food Syst. 2009, 24.

4. Mäder, P.; Pfiffner, L.; Fließbach, A.; Niggli, U. Biodiversity and Land Use: The Role of Organic Farming. In Biodiversity and Land Use: The Role of Organic Farming; Isart, J., Llerena, J., Eds.; Multitext: Barcelona, 1995; pp. 45-57 ISBN 849215330X.

5. Mäder, P.; Fließbach, A.; Dubois, D.; Gunst, L.; Fried, P.; Niggli, U. Soil fertility and biodiversity in organic farming. Science (80-. ). 2002, 296, doi:10.1126/science.1071148.

6. Carpenter-Boggs, L.; Reganold, J.P.; Kennedy, A.C. Biodynamic preparations: Short-term effects on crops, soils, and weed populations. Am. J. Altern. Agric. 2000, 15, doi:10.1017/S0889189300008614.

7. Davidson, E.A.; Galloway, L.F.; Strand, M.K. Assessing available carbon: Comparison of techniques across selected forest soils. Commun. Soil Sci. Plant Anal. 1987, 18, doi:10.1080/00103628709367802.

8. Burkitt, L.L.; Small, D.R.; McDonald, J.W.; Wales, W.J.; Jenkin, M.L. Comparing irrigated biodynamic and conventionally managed dairy farms. 1. Soil and pasture properties. Aust. J. Exp. Agric. 2007, 47, doi:10.1071/EA05196.

9. Burkitt, L.L.; Wales, W.J.; McDonald, J.W.; Small, D.R.; Jenkin, M.L. Comparing irrigated biodynamic and conventionally managed dairy farms. 2. Milk production and composition and animal health. Aust. J. Exp. Agric. 2007, 47, doi:10.1071/EA06085.

10. Longa, C.M.O.; Nicola, L.; Antonielli, L.; Mescalchin, E.; Zanzotti, R.; Turco, E.; Pertot, I. Soil microbiota respond to green manure in organic vineyards. J. Appl. Microbiol. 2017, 123, doi:10.1111/jam.13606.

11. Tassoni, A.; Tango, N.; Ferri, M. Comparison of biogenic amine and polyphenol profiles of grape berries and wines obtained following conventional, organic and biodynamic agricultural and oenological practices. Food Chem. 2013, 139, doi:10.1016/j.foodchem.2013.01.041.

12. Chalker-Scott, L. The science behind biodynamic preparations: A literature review. Horttechnology $2013,23$.

13. Spaccini, R.; Mazzei, P.; Squartini, A.; Giannattasio, M.; Piccolo, A. Molecular properties of a fermented manure preparation used as field spray in biodynamic agriculture. Environ. Sci. Pollut. Res. 2012, 19, doi:10.1007/s11356-012-1022-x.

14. Giannattasio, M.; Vendramin, E.; Fornasier, F.; Alberghini, S.; Zanardo, M.; Stellin, F.; Concheri, G.; Stevanato, P.; Ertani, A.; Nardi, S.; et al. Microbiological features and bioactivity of a fermented manure product (Preparation 500) used in biodynamic agriculture. J. Microbiol. Biotechnol. 2013, 23, doi:10.4014/jmb.1212.12004.

15. D'Haeze, W.; Mergaert, P.; Promé, J.C.; Holsters, M. Nod factor requirements for efficient stem and root nodulation of the tropical legume Sesbania rostrata. J. Biol. Chem. 2000, 275, doi:10.1074/jbc.275.21.15676.

16. Schumann, P.; Stackebrandt, E.; Goodfellow, M. Nucleic Acid Techniques in Bacterial Systematics (Modern Microbiological Methods). XXIX; Schumann, P., Stackebrandt, E., Goodfellow, M., Eds.; John Wiley \& Sons: New York - Brisbane - Toronto - Singapore, 1991;

17. Weisburg, W.G.; Barns, S.M.; Pelletier, D.A.; Lane, D.J. 16 S ribosomal DNA amplification for phylogenetic study. J. Bacteriol. 1991, 173, doi:10.1128/jb.173.2.697-703.1991.

18. White, T.J.; Bruns, T.; Lee, S.; Taylor, J. Amplification and direct sequencing of fungal ribosomal RNA genes for phylogenetics. In PCR Protocols; 1990.

19. Quince, C.; Lanzén, A.; Curtis, T.P.; Davenport, R.J.; Hall, N.; Head, I.M.; Read, L.F.; Sloan, W.T. Accurate determination of microbial diversity from 454 pyrosequencing data. Nat. Methods 2009, 6, doi:10.1038/nmeth.1361.

20. Caporaso, J.G.; Kuczynski, J.; Stombaugh, J.; Bittinger, K.; Bushman, F.D.; Costello, E.K.; Fierer, N.; Pẽa, A.G.; Goodrich, J.K.; Gordon, J.I.; et al. QIIME allows analysis of high-throughput community sequencing data. Nat. Methods 2010, 7.

21. Kõljalg, U.; Nilsson, R.H.; Abarenkov, K.; Tedersoo, L.; Taylor, A.F.S.; Bahram, M.; Bates, S.T.; Bruns, T.D.; Bengtsson-Palme, J.; Callaghan, T.M.; et al. Towards a unified paradigm for sequence-based identification of fungi. Mol. Ecol. 2013, 22. 
22. Shanks, O.C.; Kelty, C.A.; Archibeque, S.; Jenkins, M.; Newton, R.J.; McLellan, S.L.; Huse, S.M.; Sogin, M.L. Community structures of fecal bacteria in cattle from different animal feeding operations. Appl. Environ. Microbiol. 2011, 77, doi:10.1128/AEM.02988-10.

23. Liu, J. hua; Zhang, M. ling; Zhang, R. yang; Zhu, W. yun; Mao, S. yong Comparative studies of the composition of bacterial microbiota associated with the ruminal content, ruminal epithelium and in the faeces of lactating dairy cows. Microb. Biotechnol. 2016, 9, doi:10.1111/1751-7915.12345.

24. Vanbroekhoven, K.; Ryngaert, A.; Wattiau, P.; De Mot, R.; Springael, D. Acinetobacter diversity in environmental samples assessed by 16S rRNA gene PCR-DGGE fingerprinting. FEMS Microbiol. Ecol. 2004, 50, doi:10.1016/j.femsec.2004.05.007.

25. Tóth, E.M.; Borsodi, A.K. The family Nocardioidaceae. In The Prokaryotes: Actinobacteria; 2014.

26. Wong, K.; Shaw, T.I.; Oladeinde, A.; Glenn, T.C.; Oakley, B.; Molina, M. Rapid microbiome changes in freshly deposited cow feces under field conditions. Front. Microbiol. 2016, 7, doi:10.3389/fmicb.2016.00500.

27. Guarro, J.; Summerbell, R.; Samson, R. Onygenales: The dermatophytes, dimorphics and keratin degraders in their evolutionary context. Studies in Mycology No. 47.; Centraalbureau voor Schimmelcultures: Utrecht, 2003; ISBN 907035148 X.

28. Alexopoulos, C.J.; Mims, C.W.; Blackwell, M. Introductory Mycology. 4th Edition. Wiley, New York. 1996, doi:10.2105/AJPH.43.6_Pt_1.781-a.

29. Coch, R.; Leube, R. Intermediate Filaments and Polarization in the Intestinal Epithelium. Cells 2016, 5, doi:10.3390/cells5030032.

30. Doveri, F.; Pecchia, S.; Vergara, M.; Sarrocco, S.; Vannacci, G. A comparative study of Neogymnomyces virgineus, a new keratinolytic species from dung, and its relationships with the Onygenales. Fungal Divers. 2012, 52, doi:10.1007/s13225-011-0120-2.

31. De Hoog, G.S.; Göttlich, E.; Platas, G.; Genilloud, O.; Leotta, G.; Van Brummelen, J. Evolution, taxonomy and ecology of the genus Thelebolus in Antarctica. Stud. Mycol. 2005, 51.

32. Bauer, R.; Oberwinkler, F.; Kalman, K. Ultrastructural markers and systematics in smut fungi and allied taxa. Can. J. Bot. 1997, 75, doi:10.1139/b97-842.

33. Lumbsch, H.T.; Huhndorf, S.M. Myconet Volume 14. Part One. Outline of Ascomycota-2009. Part Two. Notes on Ascomycete Systematics. Nos. 4751-5113. Fieldiana Life Earth Sci. 2010, 1, doi:10.3158/1557.1.

34. Redhead, S.A.; Vilgalys, R.; Moncalvo, J.M.; Johnson, J.; Hopple, J. Coprinus Pers. and the disposition of Coprinus species sensu lato. Taxon 2001, 50.

35. Kirk, P.M.; Cannon, P.F.; Stalpers, J.; Minter, D.W. Dictionary of the Fungi 10th ed.-CABI (2008)2; 2008;

36. Brinton, W. Dynamic chemical processes underlying BD horn manure (500) preparation. J. Biodyn. 1997, 214, 1-8.

37. Madigan, M.; Cox, S.S.; Stegeman, R.A. Nitrogen fixation and nitrogenase activities in members of the family Rhodospirillaceae. J. Bacteriol. 1984, 157, doi:10.1128/jb.157.1.73-78.1984. 